\title{
Potentiometric characterisation of cyclodextrin inclusion complexes of local anaesthetics
}

Isabel Brandariz $\mathrm{a}^{*} \&$ Emilia Iglesias ${ }^{\mathrm{a}}$

${ }^{a}$ Departamento de Química Física e E. Q. I Facultad de Ciencias, Universidad de A Coruña , 15071-A , Coruña , Spain

Supramolecular Chemistry, Volume 23, Issue 9, 2011, pages 607-613

Received: 10 Mar 2011, Accepted: 6 May 2011, Published online: 05 Jul 2011

DOI: $10.1080 / 10610278.2011 .593632$

To cite this article: Isabel Brandariz \& Emilia Iglesias (2011) Potentiometric characterisation of cyclodextrin inclusion complexes of local anaesthetics, Supramolecular Chemistry, 23:9, 607-613, DOI: 10.1080/10610278.2011.593632

\section{Abstract}

The complexation of several local anaesthetics by $\beta$ and $\gamma$-cyclodextrins was studied by potentiometry with glass electrode. Tetracaine and dibucaine complexation constants were determined at $25^{\circ} \mathrm{C}$ in the presence of $0.1 \mathrm{M}$ of $\mathrm{NaCl}$. It was found that prilocaine and lidocaine complexes cannot be detected.

\section{Keywords}

potentiometric technique, local anaesthetics, inclusion complexes, cyclodextrin 


\section{Introduction}

Cyclodextrins (CDs) form inclusion complexes with a large variety of guests and act as drugdelivery systems. This ability depends on the compatibility of the guest with the size of the conical cylinder cavity of the CD and the affinity in terms of polarity with the hydrophobic wall of the cavity ( 1-3). An interesting group of compounds that form this kind of complexes are local anaesthetics that have been studied previously in our laboratory (4-8). Stability constants of procaine were obtained using a wide variety of techniques (fluorescence or UVvis spectroscopies, electrical conductivity, potentiometry and kinetic experiments) and conditions (4-7); in the same manner, tetracaine (TCA) was studied by fluorescence spectroscopy ( 8 ) and the corresponding stability constants of the complexes were determined.

This paper aims to compare those data with the same constants obtained by potentiometry for the case of TCA, with which compound both techniques can be applied, and to determine potentiometrically the stability constants of the anaesthetics, dibucaine (DCA), lidocaine (LCA) and prilocaine (PCA), the constants of which cannot be obtained by fluorescence spectroscopy, as will be discussed below.

TCA [2-(dimethylamino)ethyl 4-(butylamino)benzoate] has two amino groups; therefore, depending on the acidity of the medium it may exist as a neutral molecule, a monocation or a dication. The same is true for DCA [2-butoxy- $N$-[2-(diethylamino)ethyl]-4quinolinecarboxamide], whereas in LCA [2-(diethylamino)- $N$-(2,6-dimethylphenyl)acetamide] and PCA [N-(2-methylphenyl)-2-(propylamino)propanamide], only one amino moiety is present and there is just one acid-base equilibrium between neutral and monoprotonated molecules, see Scheme 1. It was that ( 8 ) the monocation and the neutral molecule of TCA are able to enter into the CD cavity with the unprotonated secondary amine group end first. As the aromatic ring of the drug, the hydrophobic part of the molecule, is inside the cavity, the fluorescence emission intensity is enhanced compared with that of the molecule in water; this fact was used to determine the stability constants for the complexes of $\mathrm{Tc}_{\mathrm{c}}$ and $\mathrm{HTc}^{+}$with CD. The same approach cannot be used for DCA because no appreciable variation in the fluorescence emission was observed in water and in the presence of CD, or with LCA and PCA because no fluorescence was observed in the last two cases. Taking this problem into account, we considered a potentiometric method to study the possible complexation of these anaesthetics with CDs. To apply this technique, we added a small amount of sodium chloride $(0.1 \mathrm{M})$ to all solutions to keep the ionic strength constant during the titration. 


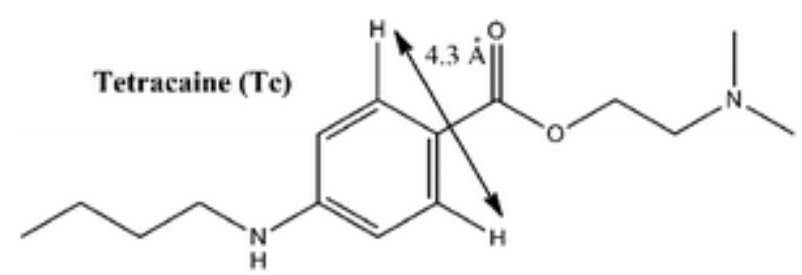

Scheme 1 Structures of the anaesthetics TCA, DCA, PCA and LCA.

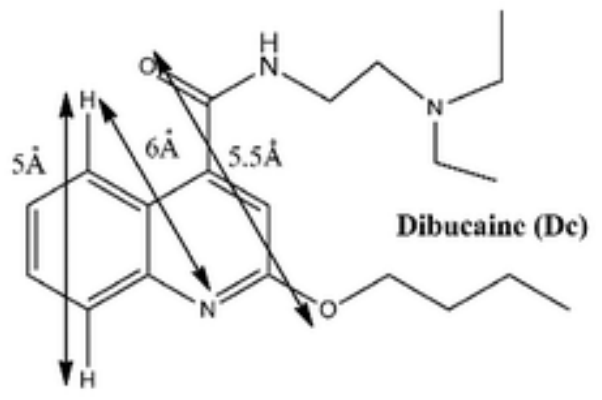

Prilocaine (Pc)

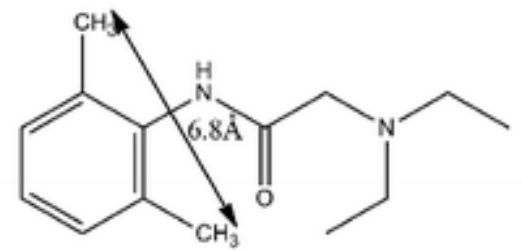

Lidocaine (Le)

If the acid-base equilibria of an anaesthetic, Xc, are represented by
(a) $\mathrm{XcH}_{2}^{2+} \stackrel{\mathrm{K}_{1}^{\prime \prime}}{\rightleftharpoons} \mathrm{XcH}^{+}+\mathrm{H}^{+}$
(b) $\mathrm{XcH}^{+} \stackrel{\mathrm{K}_{2}^{11}}{\rightleftharpoons} \mathrm{Xc}+\mathrm{H}^{+}$,

the complexation equilibrium of anaesthetic cation is, then, given by

$$
\mathrm{XcH}^{+}+\mathrm{CD} \stackrel{\mathrm{k}_{2}^{\mathrm{c}}}{\rightleftharpoons} \mathrm{XcH} \cdot \mathrm{CD}^{+}
$$

and the complexation equilibrium of neutral anaesthetic may be represented by

$$
\mathrm{Xc}+\mathrm{CD} \stackrel{\mathrm{K}_{3}^{\mathrm{C}}}{\rightleftharpoons} \mathrm{Xc} \cdot \mathrm{CD}
$$

The constants $\mathrm{K}_{2}^{\mathrm{C}}$ and $\mathrm{K}_{3}^{\mathrm{C}}$ are used in accordance with previous notation $(4,8)$, where $\mathrm{K}_{1}^{\mathrm{C}}$ represents the stability equilibrium constant of the hypothetical complex between $C D$ and the anaesthetic dication. It was also concluded that the fully protonated anaesthetic molecule does not form inclusion complexes. These equations are valid for TCA and DCA. In 
the cases of LCA and PCA, the first equilibrium, $\mathrm{K}_{1}^{\mathrm{H}}$, does not exist because there is only one amine group available to be protonated.

\section{Experimental}

High-purity TCA.HCl, DCA.HCl, LDA.HCl, PDA.HCl and $\beta-C D$ were purchased from SigmaAldrich (St Louis, MO, USA), whereas Y-CD was purchased from Cyclolab R\&D Laboratory Ltd., Budapest, Hungary.

An aqueous aliquot containing the anaesthetic hydrochloride (TCA, DCA) was titrated with a standard solution of hydrochloric acid (Merck, Westpoint, PA, USA) to determine ${ }^{p \mathrm{~K}_{1}^{\mathrm{H}}}$, and with a standard solution of sodium hydroxide (Merck) to determine ${ }^{p K_{2}^{H}}$. When CD was added to the solution of the anaesthetic hydrochloride, the titration with $\mathrm{HCl}$ yields $\log \mathrm{K}_{2}^{\mathrm{C}}$, whereas the titration with $\mathrm{NaOH}$ yields $\log \mathrm{K}_{3}^{\mathrm{C}}$. In the case of LDA. $\mathrm{HCl}$ and PDA.HCl, only titrations with base (with and without CD) were carried out because there is only one acidbase equilibrium. The anaesthetic concentration was made to vary from $10^{-2}$ to $10^{-3} \mathrm{M}$; the concentration was higher in acidic medium than in basic medium because in the latter medium the anaesthetic is in the form of a neutral molecule that easily precipitates. This is especially true for DCA, the concentration of which was kept as low as $4.5 \cdot 10^{-5} \mathrm{M}$ in basic media to avoid precipitation. Under these conditions, $0.01 \mathrm{M} \mathrm{NaOH}$ was used as a titrant which was freshly prepared before use with water bubbled with nitrogen. On the other hand, the necessary amount of $\mathrm{NaCl}$ (Merck) to adjust the ionic strength, $I$, to the desired value was added to all solutions $(I=0.1 \mathrm{M})$.

Titrations were carried out in a dual-wall cell, kept at a constant temperature $\left(25.0^{\circ} \mathrm{C}\right)$ by a thermostat-controlled system. Purified nitrogen was bubbled through the solutions to ensure thorough homogenisation and $\mathrm{CO}_{2}$ removal. A Crison microBu 2030 automatic burette furnished with a $2.5 \mathrm{ml}$ syringe for dispensing the titrant was used. The burette was controlled with a computer that was used to read the emf values from a Crison micropH 2000 $\mathrm{pH}$ meter connected to two electrodes: a glass electrode (Radiometer pHG211) and a reference electrode (Radiometer REF201).

Equilibrium constants were calculated from the potentiometric titration data by means of the Hyperquad program (9). The electrode response at a constant ionic strength is given by $E=E^{0^{\prime}}+s \log \left[\mathrm{H}^{+}\right]$, where $E$ is the emf, and the electrode parameters, the formal potential $E^{0^{\prime}}$, and the slope $s$, are required by the program to determine the constants. $A$ separate experiment was carried out to obtain the electrode parameters: emf was measured in solutions of known proton concentration at the desired ionic strength, and $s$ and $E^{0^{\prime}}$ were 
obtained from a linear regression, see Ref. (10). Measurements in proton concentration, $\left[\mathrm{H}^{+}\right]$, allow the determination of stoichiometric or concentration constants. The values of $s$ and $E^{0^{\prime}}$ for the corresponding electrode were used to plot $p[\mathrm{H}]=-\log \left[\mathrm{H}^{+}\right]=\mathrm{pH}$.

\section{Results}

\section{TCA complexes}

Acid-base constants of TCA were previously obtained by potentiometry, whereas stability constants of TCA complexes with $\beta-C D$ were determined by spectrofluorimetry; $\log \mathrm{K}_{2}^{\mathrm{C}}$ and $\log \mathrm{K}_{3}^{\mathrm{C}}$ are listed in Table 1, together with the values found in this work, for purposes of comparison. When TCA hydrochloride was dissolved in water and titrated with strong acid, an equilibrium is established between dication and cation, Equation (1a). When $\beta-C D$ was added to this system, the titration curve is displaced towards more acidic $\mathrm{pH}$ values due to the cation being taken by the $\mathrm{CD}$, Equation (2), shifting equilibrium (1a) to the right. This effect is clearly observed in Figure 1(a), in which solutions of equal concentration of TCA hydrochloride were titrated with and without CD, using the same electrode, the same day, and with the same titrant solution. The difference between both curves of the figure is proof of the complexation with $C D$, which can be used to determine the complexation constant when $p \mathrm{~K}_{1}^{\mathrm{H}}$ is known. Several titrations of TCA with CD in acid media were introduced together in Hyperquad to yield the value listed in Table1. It may be argued that these data are not of practical interest because too low a pH is needed to find them, but this is not true because TcH.CD is present at higher $\mathrm{pH}$ values too. In fact, at slightly basic $\mathrm{pH}$, Equation (1b) governs the system, and the equilibrium is established between the neutral molecule, $\mathrm{Tc}$, and the cation, $\mathrm{TcH}^{+}$; when $\beta-\mathrm{CD}$ is added to this system, it will remove both species partially to form the complexes Tc.CD and TcH.CD ${ }^{+}$, and $\log \mathrm{K}_{2}^{\mathrm{C}}$ and $\log \mathrm{K}_{3}^{\mathrm{C}}$ are needed to explain the system behaviour. In Figure $1(\mathrm{~b}), \mathrm{TCA} . \mathrm{HCl}$ is titrated with strong base, with and without $\beta-C D$, the difference between the curves is caused by the formation of the complexes, and this kind of titration can be used to determine their stability constants. At this point, two options are possible, as $\log \mathrm{K}_{2}^{\mathrm{C}}=3.00$ is known from acid titrations, this value can be fixed in the Hyperquad program to obtain the other complexation constant, $\log \mathrm{K}_{3}^{\mathrm{C}}=3.51$ (Table 1). Another choice considers both constants as unknown parameters in Hyperquad and determines both values with basic titrations. Of course, the error was higher when two constants were determined instead of only one with the same set of titrations, but the results of these two procedures agree quite well within the experimental error, Table 1. In other systems (when some problems difficult the experimental 
determination of the constants), it may be necessary to use both kinds of titrations, for example in DCA, as it will be discussed later.

Table 1 Acid-base and complexation constants of TCA and DCA at $25^{\circ} \mathrm{C}$ and $0.1 \mathrm{M}$ ionic strength of $\mathrm{NaCl}$.

\section{TCA with $\beta-C D$}

\begin{tabular}{|c|c|c|c|c|}
\hline$p \mathrm{~K}_{1}^{\mathrm{H}}$ & $p \mathrm{~K}_{2}^{\mathrm{H}}$ & $\log \mathrm{K}_{2}^{\mathrm{C}}$ & $\log \mathrm{K}_{3}^{\mathrm{C}}$ & Media \\
\hline \multirow[t]{5}{*}{$2.24^{1}$} & $8.39^{1}$ & $3.00^{1}$ & $3.53^{1}$ & \\
\hline & & $3.00 \pm 0.01$ & & Acid titration, Equations $(\underline{1 \mathrm{a}})$ and $(\underline{2})$ \\
\hline & & & $3.51 \pm 0.01$ & Basic titration, using $\log \mathrm{K}_{2}^{\mathrm{C}}=3.00$ \\
\hline & & $2.9 \pm 0.1$ & $3.48 \pm 0.07$ & Basic titration, Equations $(\underline{1 b}),(\underline{2})$ and $(\underline{3})$ \\
\hline & & $3.01 \pm 0.02$ & $3.51 \pm 0.02$ & Acid+basic titration together \\
\hline
\end{tabular}

TCA with $Y-C D$

\begin{tabular}{|c|c|c|c|c|}
\hline & & $\log \mathrm{K}_{2}^{\mathrm{C}}$ & $\log K_{3}^{C}$ & \\
\hline & & & $1.85^{1}$ & \\
\hline & & $2.41 \pm 0.05$ & $2.84 \pm 0.03$ & Acid+basic titration together \\
\hline \multicolumn{5}{|c|}{$D C A$ with $\beta-C D$} \\
\hline$p \mathrm{~K}_{2}^{\mathrm{H}}$ & $p \mathrm{~K}_{2}^{\mathrm{H}}$ & $\log \mathrm{K}_{2}^{\mathrm{C}}$ & $\log \mathrm{K}_{3}^{\mathrm{C}}$ & \\
\hline $1.95 \pm 0.01$ & $8.77 \pm 0.01$ & $2.61 \pm 0.02$ & $2.65 \pm 0.02$ & Acid+basic titration together \\
\hline \multicolumn{5}{|c|}{$D C A$ with $\gamma-C D$} \\
\hline & & $\log \mathrm{K}_{2}^{\mathrm{C}}$ & $\log \mathrm{K}_{3}^{\mathrm{C}}$ & \\
\hline & & $2.28 \pm 0.04$ & $2.58 \pm 0.03$ & Acid+basic titration together \\
\hline
\end{tabular}

${ }^{1}$ Ref. ( $\underline{8}$ ). 


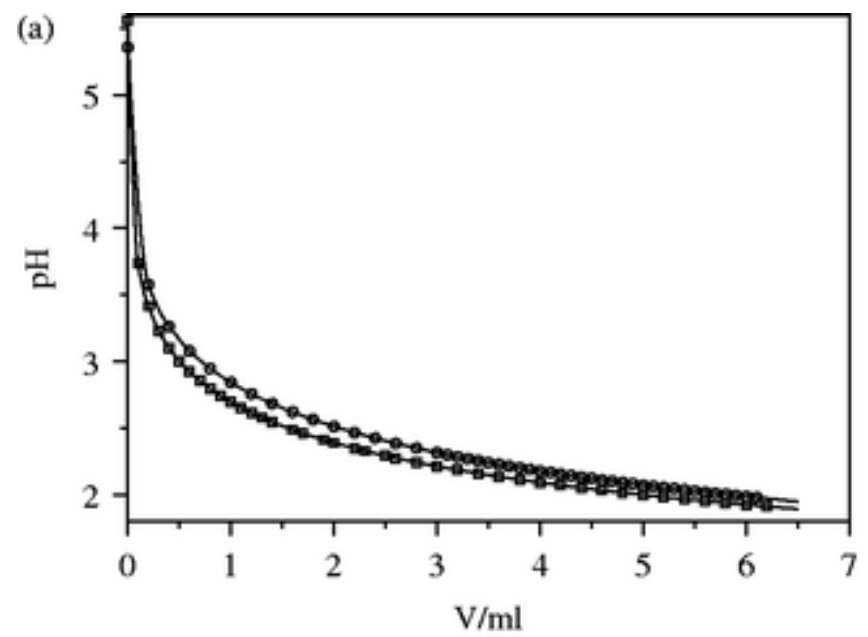

Figure 1 TCA hydrochloride titration without (circles) and with $\beta-C D$ (squares) (a) in acid media, $\quad[T C A]=3 \mathrm{mM}, \quad[\beta-\mathrm{CD}]=3 \mathrm{mM}$, $[\mathrm{HCl}]=0.1, v_{0}=40 \mathrm{ml}$ and (b) in basic media, $\quad[\mathrm{TCA}]=1 \mathrm{mM}, \quad[\beta-\mathrm{CD}]=1 \mathrm{mM}$, $[\mathrm{NaOH}]=0.010 \mathrm{M}, v_{0}=40 \mathrm{ml} \quad\left(v_{0}\right.$ is the initial volume of titrand).

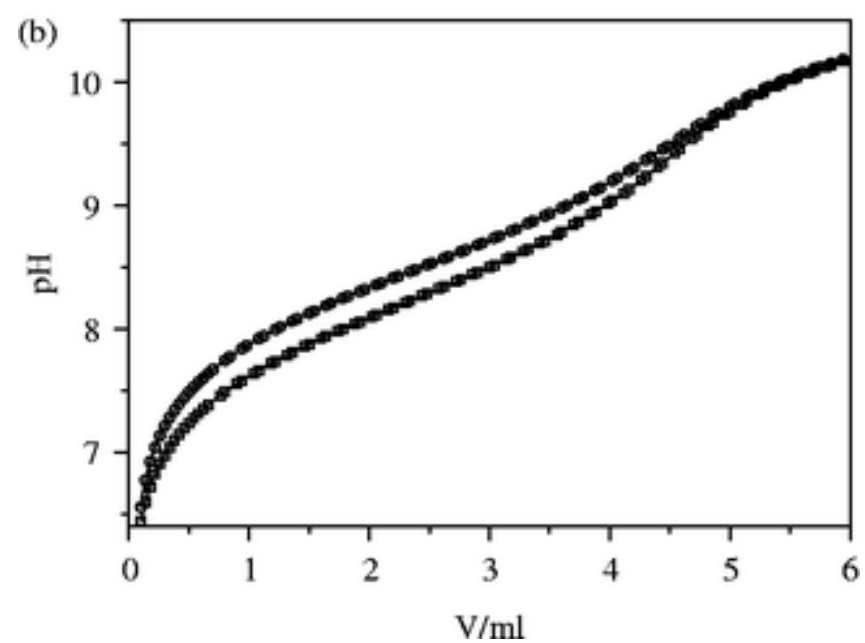

Complexation constants of TCA with Y-CD are also included in Table 1. Titrations in acid and basic media were used to obtain these values. It is interesting to note that when TCA was titrated with acid in the presence of $\mathrm{Y}$-CD (four titrations), Hyperquad fitting yields $\log \mathrm{K}_{2}^{\mathrm{C}}=2.40$ and $p \mathrm{~K}_{1}^{\mathrm{H}}=2.26$; therefore, $p \mathrm{~K}_{1}^{\mathrm{H}}$ can be determined simultaneously with $\mathrm{K}_{2}^{\mathrm{C}}$ in the presence of $\mathrm{y}-\mathrm{CD}$; as it can be seen in Table 1 this result agrees perfectly with $\mathrm{pK}_{1}^{\mathrm{H}}=2.24$ obtained without $\mathrm{Y}$-CD. If the complexation equilibrium is omitted from the system, an incorrect value of $p \mathrm{~K}_{1}^{\mathrm{H}}=1.96$ was determined, which clearly shows that the presence of $\mathrm{Y}-\mathrm{CD}$ affects the acid-base equilibrium (of course, similar behaviour is observed with $\beta-C D)$.

A comparison of the data obtained in this paper with those determined previously from fluorescence measurements ( 8 ) shows that the stability constants with $\beta-C D$ agree very well, whereas $\mathrm{K}_{3}^{\mathrm{C}}$ with $\mathrm{y}-\mathrm{CD}$, obtained in this work, is significantly higher. The explanation may reside in the fact that the concentration of TCA in fluorescence experiments was quite low $\sim 10 \mu \mathrm{M}$ (it was selected taking into account that the absorbance should be $\sim 0.2$ ). But 
buffer, $\mathrm{HCO}_{3}^{-} / \mathrm{CO}_{3}^{2-}$, was added in a concentration of $0.033 \mathrm{M}$ to adjust the $\mathrm{pH}$, then it was in a much larger quantity than TCA and it could be able to compete with the anaesthetic to form complexes with $\mathrm{Y}-\mathrm{CD}$. This result emphasises the interest of using different methods to study the complex formation.

\section{DCA complexes}

It was mentioned in the experimental section that the neutral molecule of DCA is less soluble than the other anaesthetics used in this study; solutions having concentrations as low as $4.5 \cdot 10^{-5} \mathrm{M}$ were used to avoid precipitation in basic media (when CD was present, $10^{-4} \mathrm{M}$ concentration solutions were used). These concentrations are quite low for the potentiometric technique ( 11 ); for this reason, titrations in acid media were used to determine $\log _{2}^{C}$. With this value and the results of the titrations in basic media, $\log \mathrm{K}_{3}^{\mathrm{C}}$ was obtained (Table 1). When only basic titrations were used in Hyperquad to obtain $\log \mathrm{K}_{3}^{\mathrm{C}}$ and $\log \mathrm{K}_{2}^{\mathrm{C}}$, the program was not able to optimise the values of both the constants simultaneously, although it was able to optimise one of them if the other was known. The reason is probably not only the low concentration of reactants, but also the close values of the constants 2.61 and 2.65, which cause no shift in the titration curves when CD was added to the system, as is explained below. It should be kept in mind that in basic media there is an equilibrium between neutral molecule, Dc, and cation, $\mathrm{DcH}^{+}$, Equation (1b). So, it is possible to calculate the complexation constants of these species, because when $C D$ was added to the solution, it removes part of these molecules to form the complexes; but if the constants are quite similar, no shift in the equilibrium is expected: the system does not detect the presence of CD. To illustrate this point, are carried out a simulation and the result is shown in Figure 2. The HySS program ( 12 ) was used to reproduce the expected experimental titration curves for $\mathrm{DCA}$ in the absence (circles) and in the presence of $\beta-C D$ (lines from a to $\mathrm{g}$ ) for different values of $\log \mathrm{K}_{3}^{\mathrm{C}}$. In this simulation process, the $\log \mathrm{K}_{2}^{\mathrm{C}}=2.60$ was kept constant, whereas $\log \mathrm{K}_{3}^{\mathrm{C}}$ was varied with the following values (a) 2.60 , (b) $2.70, \ldots$, (g) 3.10 . The concentrations used to obtain Figure $2(\mathrm{a})$ were $[\mathrm{DCA}]=1.5 \cdot 10^{-4} \mathrm{M}$ and $[\beta-C D]=1.5 \cdot 10^{-3} \mathrm{M}$ titrated with $[\mathrm{NaOH}]=0.01 \mathrm{M}$. In Figure $2(\mathrm{~b})$, these values were increased 10 times to illustrate the influence of concentration, i.e. $[D C A]=1.5 \cdot 10^{-3} \mathrm{M}$ and $[\beta-C D]=1.5 \cdot 10^{-2} \mathrm{M}$ titrated with $[\mathrm{NaOH}]=0.1 \mathrm{M}$. Several remarkable facts can be observed when these graphs are compared. The most evident point is that at high concentrations all features of the curves are more clearly observed: $\mathrm{pH}$ variation and separation among curves are larger (notice that $\mathrm{pH}$ scale is the same in both plots). It is a good way to show the difficulties that appear when 
low concentrations are used, as it was stated before. Besides, this is the reason why an effect that is almost imperceptible in Figure 2(a) becomes clearly observable in Figure 2(b), i.e. the separation of the curves, at high $\mathrm{pH}$, when $\beta-\mathrm{CD}$ (curves $\mathrm{a}-\mathrm{g}$ ) is present and when the anaesthetic is titrated alone (circles). The explanation is quite simple, the acid-base equilibrium of $\beta-C D$ was taken into account in the simulations, and its influence can be noticed at the end of the titrations. It should be noted that $\beta-C D$ concentration is higher than that of the anaesthetic, not only in the simulation, but in the experimental titrations too, in which $\beta-C D$ concentration used was equal or several times higher than that of the anaesthetic. As this effect was observed in the experimental data, the determination of the acid-base constants of the CDs used in this work is interesting and it will be discussed later. Although there is a separation of the curves at high $\mathrm{pH}$, because of the reason explained above, at lower $\mathrm{pH}$, when both constants are equal, the plots coincide very well, curves $a$ and circles Figure 2(a,b), whereas the other curves $b-g$ increase their separation with respect to the circles as long as the difference between $\log \mathrm{K}_{3}^{\mathrm{C}}$ and $\log \mathrm{K}_{2}^{\mathrm{C}}$ increases. Then, if both complexation constants are equal, titration curves of the anaesthetic remain almost the same in the presence and absence of $\mathrm{CD}$, unless at high $\mathrm{pH}$, in which $\mathrm{pK}$ of $\mathrm{CD}$ becomes relevant, but this is the same behaviour that would be expected if CD would not form complexes at all with the anaesthetic. This is not a problem when one of the constants can be found by other means, in this case the acid titrations yield the complexation constant of the cation and when this value is known, titrations with base yield the complexation constant of the neutral molecule. The values of the constants in this work agree well with others found in the literature $(13),{ }^{p K_{2}^{H}}=8.73$, and ${ }^{\log K_{3}^{C}}=2.8$, with $\beta-C D$, using drugsensitive electrodes ( 14 ). 

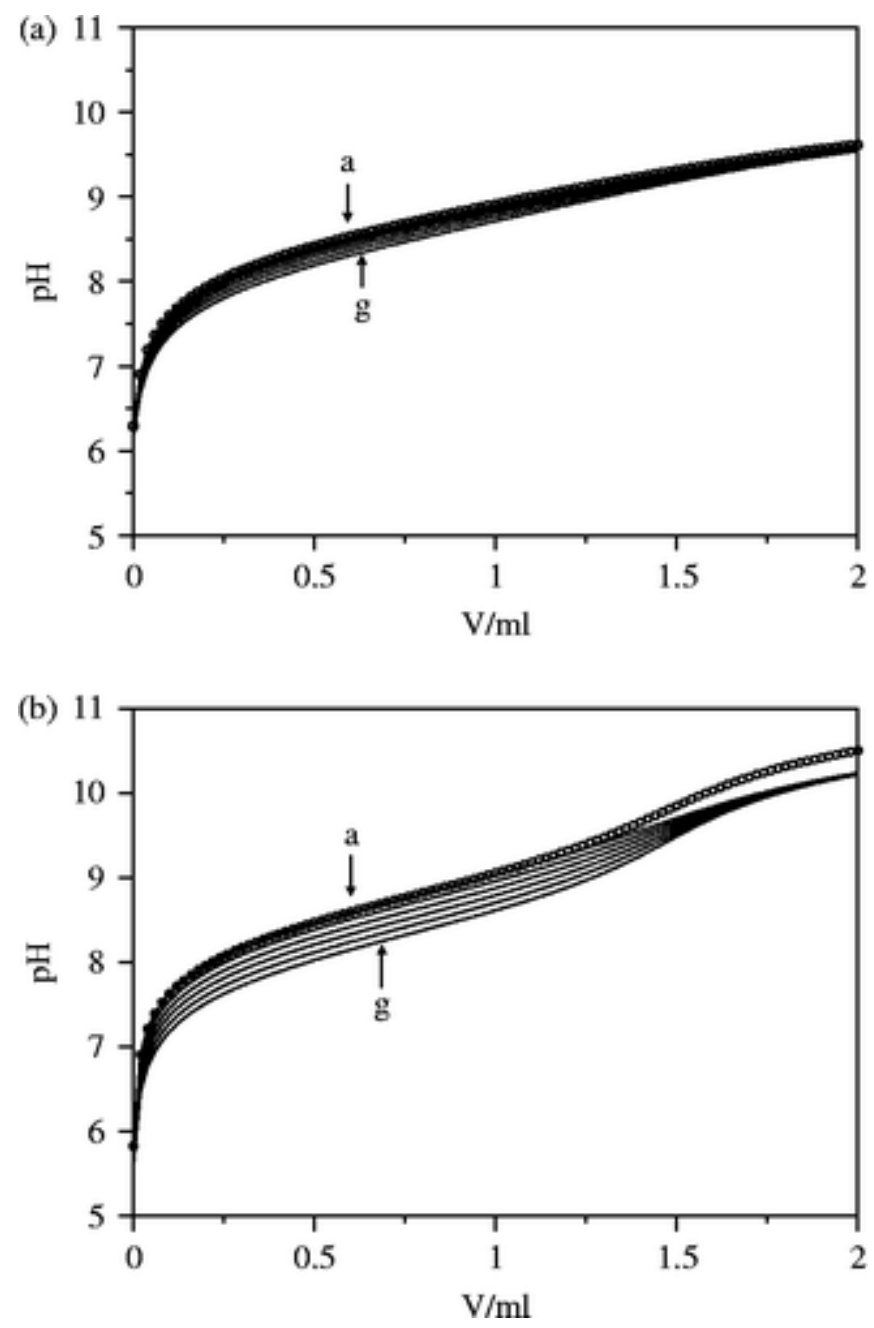

Figure 2 (a) Simulation of the expected titration curves for DCA in the absence (circles) and in the presence of $\beta-C D$ (lines) with constants: $p \mathrm{~K}_{1}^{\mathrm{H}}=1.95, p \mathrm{~K}_{2}^{\mathrm{H}}=8.77$ $p \mathrm{~K}_{\beta \cdot \mathrm{CD}}^{\mathrm{H}}=11.95$

, $\log K_{2}^{C}=2.60$ and $\log K_{3}^{C}$ changing with the following values $2.60,2.70,2.80,2.90$, 3.10 and 3.10 (lines $a-g$ ); concentrations are $\quad[D C A]=1.5 \cdot 10^{-4} \mathrm{M}$ and $[\beta$ $C D]=1.5 \cdot 10^{-3} \mathrm{M} \quad$ titrated with $[\mathrm{NaOH}]=0.01 \mathrm{M}, v_{0}=100 \mathrm{ml}$.

Simulation of the expected titration curves for DCA under the same conditions of Figure 2(a), except that concentrations were increased 10 times: $[\mathrm{DCA}]=1.5 \cdot 10^{-3} \mathrm{M}$ and $[\beta-C D]=1.5 \cdot 10^{-2} \mathrm{M} \quad$ titrated with $[\mathrm{NaOH}]=0.1 \mathrm{M}$.

\section{LCA and PCA complexes}

LCA and PCA have only one amino group and only one acid-base equilibrium, Equation (1b). Titration with base of the PCA hydrochloride alone, with $\beta-C D$ and $y-C D$, is presented in Figure 3. The concentration of the anaesthetic was the same in the three experiments that were carried out using the same electrode, the same day and with the same titrant solution. As it can be appreciated, all the curves coincide quite well except at high $\mathrm{pH}$, in which the influence of the acid-base equilibrium of the CD is appreciable; the same behaviour can be observed in Figure 2(b). At the end of titration, PCA was converted from the protonated to the neutral form, while $C D$ begins to ionise causing the separation of the curves. Similar behaviour was observed for LCA. Several titrations in the presence of CD ( $\beta-C D$ or $y-C D)$ were fitted with Hyperquad by fixing the $p K$ of $C D$ (Table 2), the $p K_{H}$ of the anaesthetic was obtained and the results are listed in Table 2, and it agrees with the literature reports $(13,15)$. Titration curves and $p \mathrm{~K}_{\mathrm{H}}$ values are equal in the presence and in the absence of $\mathrm{CD}$; therefore, it is impossible to determine the stability constant of the inclusion complexes 
because no separation in the curves appears. There are various possible explanations. One of them is evident and may be attributed to the absence of complex formation; on the other hand, a more subtle explanation is that complexes were formed, but the stability constants are approximately equal and no variation is expected in the curves (see Figure 2) or even the anaesthetic does not penetrate very deeply in the cavity of the CD and no disturbance in the acid-base equilibrium was observed. Previously ( 16 ), inclusion complexes of the neutral molecules of some anaesthetics, including PCA and LCA, with $\beta-C D$ were determined from the solubility measurements. Quite low values for the stability constants were obtained such as $\mathrm{K}_{3}^{\mathrm{C}}=26 \mathrm{M}^{-1}$ for LCA and $\mathrm{K}_{3}^{\mathrm{C}}=96 \mathrm{M}^{-1}$ for PCA. To illustrate the expected shifts in the titration curves that would be originated by such low-complexation constants, we carried out a new simulation with the HySS program ( 12 ). The results are shown in Figure 3(b), when the concentrations used were the same as those appearing in the experimental titrations of Figure 3(a) for the case of $P C A$, i.e. $40 \mathrm{ml}$ of $[P C A]=1.0 \cdot 10^{-3} \mathrm{M}$ and $[\beta-C D]=4.9 \cdot 10^{-3} \mathrm{M}$ titrated with $[\mathrm{NaOH}]=0.02 \mathrm{M}$, whereas the constants used were $\mathrm{pK}^{\mathrm{H}}=8.01$ , $\log \mathrm{K}_{3}^{\mathrm{C}}=\log (96)=1.98$ was kept constant, and $\log \mathrm{K}_{2}^{\mathrm{C}}$ was changed with the following values (a) 1.98 , (b) $1.88, \ldots$, (g) 1.58 . The new fact in this plot, compared with the other simulations, is that the curves are very close to one another, showing the difficulties that appear when the constants are too low, which seems to be the case here, and, of course, this precludes the potentiometric determination of the constants. 

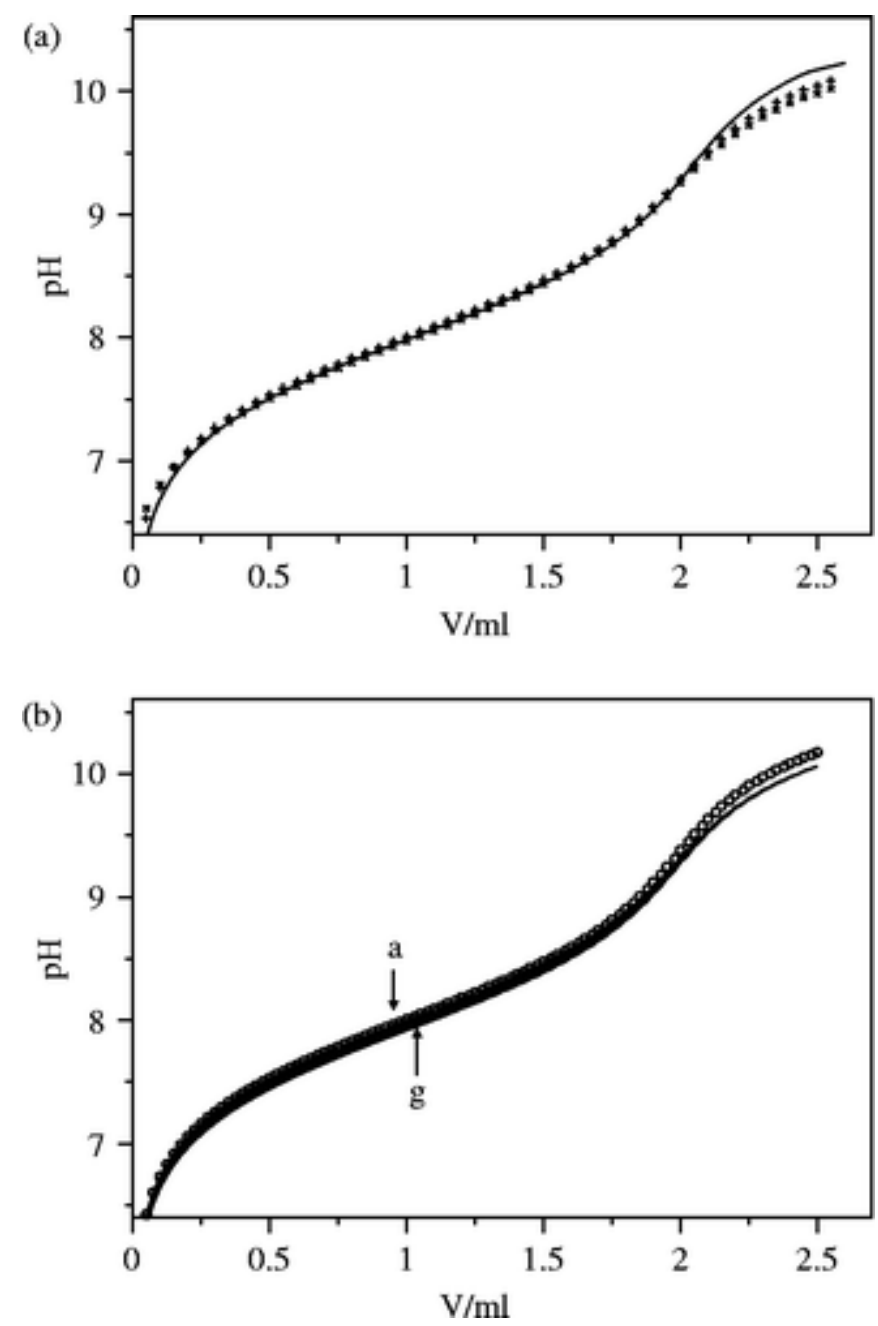

Figure 3 (a) PCA hydrochloride titration alone (line), with $\beta-C D(+)$ and with $\gamma-C D$ $(x), \quad[P C A]=1 \mathrm{mM}, \quad[\beta-C D]=5 \mathrm{mM}, \quad\left[\gamma^{-}\right.$ $\mathrm{CD}]=6 \mathrm{mM}, \quad[\mathrm{NaOH}]=0.02 \mathrm{M}, v_{0}=40 \mathrm{ml}$. (b) Simulation of the expected titration curves of $40 \mathrm{ml}$ of PCA with concentration $1 \mathrm{mM}$ and $[\beta-C D]=4.9 \mathrm{mM}$ titrated with $[\mathrm{NaOH}]=0.02 \mathrm{M}$, the constants are $p \mathrm{~K}^{\mathrm{H}}=8.01$ , $\log \mathrm{K}_{3}^{\mathrm{C}}=1.98$ and $\log \mathrm{K}_{2}^{\mathrm{C}}$ changing with the following values $1.98,1.88,1.78,1.68$ and 1.58 (lines $a-g$ ), circles correspond to the titration in the absence of $C D$.

Table 2 Acid-base constants of LCA, PCA, $\beta-C D$ and $y-C D$ at $25^{\circ} \mathrm{C}$ and $0.1 \mathrm{M}$ ionic strength of $\mathrm{NaCl}$

\begin{tabular}{lc}
\hline & $p \mathrm{~K}^{\mathrm{H}}$ \\
\hline LCA & $7.92 \pm 0.01$ \\
LCA with $\beta-C D$ & $7.89 \pm 0.01$ \\
LCA with Y-CD & $7.90 \pm 0.01$ \\
PCA & $8.01 \pm 0.01$ \\
PCA with $\beta-C D$ & $8.03 \pm 0.01$ \\
PCA with $Y-C D$ & $7.99 \pm 0.01$ \\
$\beta-C D$ & $11.95 \pm 0.01$ \\
Y-CD & $11.88 \pm 0.02$
\end{tabular}




\section{Acid-base constants of $\beta-C D$ and $Y-C D$}

As it has been discussed before, the last points of the titration curves in basic media are influenced by the presence of CD. This fact encouraged us to estimate the $p K$ of $\beta$ - and $y$ CDs, under the experimental conditions of this work, $25^{\circ} \mathrm{C}$ and $I=0.10 \mathrm{M} \mathrm{NaCl}$. The results are listed in Table 2. Obviously with these $\mathrm{pK}$ values, high $\mathrm{pH}$ (more than 12) has to be reached in the titrations, which is inconvenient for the potentiometric technique (11). Besides, in this situation the ionisation constant of water has to be considered, a value of $p \mathrm{~K}_{w}^{\mathrm{C}}=13.79$ at $25^{\circ} \mathrm{C}$ and $I=0.10 \mathrm{M} \mathrm{NaCl}$ was used (17). If $p \mathrm{~K}_{w}$ is not fixed in the fitting program and is determined together with the $p K$ of the $C D$, the obtained results were 13.77 for $\beta-C D$ and 13.79 for $\gamma-C D$, which agree very well with the values found using a hydrogen electrode ( 17$)$. On the other hand, slightly higher values for the $p K$ of both CDs (0.3 units more) can be found in the literature (18). This difference can be ascribed to the experimental error at high $\mathrm{pH}$ and besides the calibration procedure that is quite different from ours. Previously ( 18 ), calibration of the electrode was carried out using standard buffers of known $\mathrm{pH}$, after that $\mathrm{pH}$ was measured and the proton concentration was obtained, using the proton activity coefficient, calculated from Debye-Hückel equation with a certain value of the size parameter in this equation. In fact, it is not necessary to have any supposition about activity or activity coefficients of individual ions to perform a calibration in concentration ( 19 ). Solutions of known concentration can be used to obtain a calibration line without any kind of supposition that could affect the obtained values in an indeterminate way.

\section{Discussion}

CDs are truncated cone-shaped molecules with a hollow, tapered cavity of $7.9-\AA$ depth. The top and bottom diameters of the cavity are 6.8 and $7.8 \AA$ for $\beta-C D$, and 8.3 and $9.5 \AA$ for $\gamma^{-}$ CD, respectively $(1)$.

As it was stated before, the ability to form inclusion complexes depends on the compatibility of the guest molecule with the size of the cavity and the affinity in terms of polarity with the hydrophobic wall of the cavity. To explore the possible inclusion of the anaesthetic molecules into the $C D$, we optimised the size of these compounds using PM6 method by means of MOPAC ( 20 ) (identical results were found with PM3). It is expected that the molecule will enter with its more hydrophobic part oriented towards the cavity, that is, the aromatic ring. Some relevant distances are depicted in Scheme 1. It can be shown that TCA has a size perfectly compatible with the cavity of CDs and gives rise to the highest constants. On the 
other hand, LCA is the molecule with the largest diameter because of the methyl groups in the benzene ring, which fact makes it difficult for the complexation with $\beta-C D$, but not with $\gamma$ $\mathrm{CD}$ that has the biggest cavity. Therefore, the absence or the small amount of complex formation may be ascribed to a lack of affinity with the hydrophobic cavity of the CD. The same seems to be true for PCA that has a smaller size than LCA. Both compounds exhibit a much higher solubility in water than DCA or TCA, and it seems they prefer the polar environment of water instead of the hydrophobic cavity of the CDs. On the contrary, DCA with a large aromatic part, even relatively big, can form complexes in significant amount. A detailed study of the geometry of these molecules, neutral, protonated and in different solvents can be found in Ref. ( 21 ).

\section{Conclusions}

The use of the potentiometric technique to determine inclusion constants of several local anaesthetics with CDs has been discussed and the complexation constants were determined. It was found that TCA, neutral or protonated, forms complexes in significant amount with $\beta$ and $\gamma$-CDs. This system had been studied previously with similar results, in this laboratory, using fluorescence. Although this is a very sensible technique, there are other anaesthetics that cannot be studied using this method, i.e. no fluorescence or change of fluorescence is observed in the presence of $C D$, so potentiometry was used. This is the case of DCA that forms complexes with both CDs; also of PCA and LCA, which do not form complexes or do it in a small amount that cannot be detected with the potentiometric technique. On the other hand, although the sizes of the cavities of the CDs are adequate to include the molecules of the anaesthetics used in this study (with the exception of LCA in $\beta$ $\mathrm{CD}$ ), the absence of complexation can be ascribed to different affinities of the guest molecule and the cavity.

\section{Acknowledgements}

Financial support from the Direccion General de Investigacion (Ministerio de Educacion y Ciencia) of Spain and FEDER (Project CTQ2005-07428/BQU) and from the Direccion General de Programas y Transferencia de Conocimiento (Ministerio de Ciencia e Innovacion) of Spain (Project CTQ2008-04429/BQU) is gratefully acknowledged.

\section{References}


1. Szejtli, J. 1998. Chem. Rev., 98: 1743-1753.

2. Rekharsky, M.V. and Inoue, Y. 1998. Chem. Rev., 98: 1875-1917.

3. Dodziuk, H., ed. 2006. Cyclodextrins and their Complexes. Chemistry, Analytical Methods and Applications, Weinheim: Wiley-VCH.

4. Iglesias, E. 2006. J. Org. Chem., 71: 4383-4392.

5. Iglesias-Martínez, E., Brandariz, I. and Penedo, F. 2006. Chem. Res. Toxicol., 19: 594-600.

6. Iglesias-Martínez, E., Brandariz, I. and Penedo, F. 2007. J. Incl. Phenom. Macrocycl. Chem., 57: 573-576.

7. Brandariz, I. and Iglesias-Martínez, E. 2009. J. Chem. Eng. Data, 54: 2103-2105.

8. Iglesias-García, I., Brandariz, I. and Iglesias, E. 2010. Supramol. Chem., 22: 228-236.

9. Gans, P., Sabatini, A. and Vacca, A. 1996. Talanta, 43: 1739-1753.

10. Brandariz, I., Barriada, J.L., Vilarino, T. and Sastre de Vicente, M.E. 2004. Monatsh. Chem., 135: 1475-1488.

11. Albert, A. and Serjeant, E.P. 1984. The Determination of lonization Constants: $A$ Laboratory Manual, 3rd, London, New York: Chapman \& Hall.

12. Alderighi, L., Gans, P., lenco, A., Peters, D., Sabatini, A. and Vacca, A. 1999. Coord. Chem. Rev., 184: 311-318.

13. Kamaya, H., Hayes, J.J. and Ueda, I. 1983. Anesth. Analg., 62: 1025-1030.

14. Takisawa, N., Shirahama, K. and Tanaka, I. 1993. Colloid Polym. Sci., 271: 499-506.

15. Levy, R.H. and Rowland, M. 1972. J. Pharm. Pharmacol., 24: 841-847.

16. Dollo, G., LeCorre, P., Chevanne, F. and LeVerge, R. 1996. Int. J. Pharmaceut., 136: 165-174.

17. Harned, H.S. and Owen, B.B. 1958. The Physical Chemistry of Electrolytic Solutions, 3rd, New York: Reinhold Publishing Coorporation.

18. Gelb, R.I., Schwartz, L.M., Bradshaw, J.J. and Laufer, A. 1980. Bioorganic Chem., 9: 299-304.

19. Mesmer, R.E. 1991. Geochim. Cosmochim. Acta, 55: 1175-1176.

20. MOPAC2007, James J.P. Stewart. 2007. Computational Chemistry, CO, USA: Colorado Springs. http://OpenMOPAC.net

21. Motta-Neto, J.D. and Alencastro, R.B. 1997. Int. J. Quantum Chem., 61: 959-980. 